\title{
Chronic corticosterone treatment enhances extinction-induced depression in aged rats
}

\author{
Joseph P. Huston ${ }^{1}$, Mara Komorowski ${ }^{1}$, Maria A. de Souza Silva ${ }^{1}$, Valéria \\ Lamounier-Zepter ${ }^{2}$, Susanne Nikolaus ${ }^{3}$, Claudia Mattern ${ }^{4}$, Christian P. Müller ${ }^{5}$ \\ Bianca Topic ${ }^{1}$
}

\footnotetext{
${ }^{1}$ Center for Behavioral Neuroscience, Institute of Experimental Psychology, Heinrich-HeineUniversity of Düsseldorf, Universitätsstr. 1, 40225 Düsseldorf, Germany

${ }^{2}$ Medical Clinic III, University of Technology Dresden, Fetscherstr. 74, 01307 Dresden, Germany

${ }^{3}$ Clinic of Nuclear Medicine, University Hospital Düsseldorf, Düsseldorf, Germany

${ }^{4} \mathrm{M}$ et P Pharma AG, 6376 Emmetten, Switzerland and Oceanographic Center, Nova Southeastern University, Fort Lauderdale, FL 33314, USA.

${ }^{5}$ Department of Psychiatry and Psychotherapy, University Clinic, Friedrich-Alexander-University of Erlangen-Nuremberg, Schwabachanlage 6, 91054 Erlangen, Germany
}

Corresponding author: Joseph P. Huston, PhD

Center for Behavioral Neuroscience

University of Duesseldorf

Universitaetsstr. 1

40225 Duesseldorf

Tel. 0049-172-2126861

FAX: +49 211-811-2024

E-mail address: Huston@uni-duesseldorf.de 


\begin{abstract}
Withdrawal and avoidance behavior are common symptoms of depression and can appear as a consequence of absence of reward, i.e. extinction-induced depression (EID). This is particularly relevant for the aged organism subjected to pronounced loss of former rewards. Avoidance of the former site of reward and increased withdrawal into a distant compartment accompany extinction of food-rewarded behavior in rodent models. During extinction, behavioral markers for re-learning dissociate from indicators of extinction-induced depression. Here we examined the effect of a chronic treatment with corticosterone (CORT), a well-known inducer of depression-related behavior, on EID in adult and aged rats. Adult (3-4 months) and aged (18 months) male rats were treated with CORT via drinking water for 3 weeks prior to extinction of a cued food-reward task. CORT treatment increased the distance from the site of reward and decreased goal tracking behavior during extinction, especially in the aged rats. Plasma hormone levels measured before and after restraint stress showed a decline in basal ACTH- and CORT-levels after chronic CORT treatment in aged animals. The treatment significantly impaired the HPA-axis activation after acute stress in both, adult and aged animals, alike. Altogether, these findings show an enhancement of EID after chronic CORT treatment in the aged organism, which may be mediated by an impaired HPA-axis sensitivity. These findings may have special relevance for the investigation of human geriatric depression.
\end{abstract}

Key words: Depression model, extinction, despair, corticosterone, ACTH, chronic stress, goal tracking 


\section{Introduction}

Loss of reward or reinforcement play a crucial role in the psychopathology of major depression and can lead to despair, anhedonia, and withdrawal (Wittchen et al., 2011), resulting in avoidance of former desirable situations (Lewinsohn, 1974). The onset of depression is often preceded by stressors and experiences of loss such as unemployment, disease or parting (Kendler et al., 1999). Experiences of loss affect particularly the aged population and may account for the increasing number of old people suffering from depression (Bruce, 2002).

The absence of formerly delivered reinforcers induces cessation, and thus, "extinction" of behavior that previously led to the reinforcer. Extinction is considered to be an active learning process, rather than passive forgetting or erasure of memories (Todd et al., 2014). Extinction of operant behavior in vertebrates has a behavioral, allocentric component, which involves learning that a particular appetitive behavior no longer leads to reward. However, extinction also has emotional, egocentric components, as the omission of an expected reward can induce emotional reactions, including frustration, despair, and aggression (Huston et al. 2013; Papini and Dudley 1997; Papini 2003). Animal models for extinction-induced depression (EID) have been developed only recently. It was shown that 'distance from the food cup' and the cessation of 'goal tracking' during extinction are sensitive to antidepressant treatment, and thus, reliable measures of EID (Huston et al. 2012, 2013, 2016; Komorowski et al. 2012). In aged rats, changes in the amount of striatal neurotransmitters (Schulz et al., 2004), neurotrophins (Topic et al., 2008a) and mineral-/glucocorticoid-receptors in the brain after extinction were reported, which correlated with EID measures upon the withholding of negative reinforcement (Topic et al., 2008b). Since especially the aged are confronted with life events that subsume loss of positive reinforcers (Kraaij et al., 2002), we have set out to examine how EID interacts with a stressor in the aged organism. In the present study, we asked how chronic corticosterone (CORT) stress affects EID during aging. We hypothesized 
that CORT treatment would exacerbate EID by increasing avoidance of the former site of reward-delivery and avoidance of goal tracking during extinction predominantly in aged rats.

\section{Materials and Methods}

\section{Subjects}

Twenty-four adult (3-4 months) and thirty aged (18 months) naive, male Wistar rats were obtained from Zentrale Einrichtung für Tierforschung und Tierschutzaufgaben (University of Düsseldorf; adults: $253.21 \pm 3.6 \mathrm{~g}$, aged: $601.93 \pm 11.86 \mathrm{~g}$ ). Water and food were provided ad libitum until food deprivation started. Animals were subjected to a $12 \mathrm{~h}$ light/dark cycle (lights on: 7am) with room temperature maintained at $22 \pm 2^{\circ} \mathrm{C}$ in the husbandry room. They were housed in type VI macrolon cages in groups of four (adult) and 2-3 (aged). After one week of familiarization, the animals were weighed, marked and food deprived (15g/animal/day for aged, 12g/animal/day for adult). The total daily fluid intake of each cage was measured during food deprivation so that the approximate amount of water intake could be calculated for each rat by division. Three handling sessions were carried out prior to the experiment. Food deprivation and weighing were continued until the end of the study. After the acquisition sessions, animals were randomly assigned to the control- or CORT-group in equal numbers ( $n=15 /$ group for aged, $n=12 /$ group for adult rats). The study was carried out in accordance with the German Law on the Protection of Animals and was approved by the state authority (Bezirksregierung Düsseldorf).

\section{Apparatus and procedure}

Elongated operant chambers with walls made of dimmed Plexiglas were used (Komorowski et al., 2012; Huston et al., 2016). The chambers were $72 \mathrm{~cm}$ long, $28 \mathrm{~cm}$ wide and $34 \mathrm{~cm}$ high (Fig. 1). The floor was covered with a black rubber mat and the top of the box was open. Each operant chamber was situated in a dark, sound attenuating box, containing a masking white 
noise of $60 \mathrm{~dB}$. Infra-red cameras (Conrad Elektronik ${ }^{\circledR}$ ) were mounted on the top and side of the chambers. The chambers were segmented by half into two compartments by additional side walls made of Plexiglas. Animals could freely enter both compartments through a transition in the middle of the walls. A triple cue light (green, yellow, red), the food magazine (BioServ ${ }^{\circledR}$ Dustless precision pellets) and the food cup (Coulbourn Industries ${ }^{\circledR}$ ) with an integrated photodetector were fastened in the middle of the short wall. A house light offered slight illumination ( 1 lux). For the magazine training sessions, an external hand-switch was connected to the apparatus to shape the animals' behavior toward the feeder. All modules were connected via a link box from PanLab® and controlled by the PackWin® 2.0.01 Software. Automated tracking of the animals' behavior was done via center-point detection and manually by an experienced rater with Ethovision XT $8 \circledR$ (Noldus $\left.{ }^{\circledR}\right)$. The sound attenuated boxes were located within the animals' husbandry room.

Three days prior to the experiment, animals received $1 \mathrm{~g}$ of the BioServ® pellets daily additionally to their restricted regular rat chow. On the first day of the experiment, all animals underwent a habituation session of 15 minutes and could freely explore the chamber. Two magazine training sessions of 15 minutes were performed on the consecutive two days (Komorowski et al., 2012). Hereby, the cue lights were turned on for five seconds each time when reinforcement was delivered. Within the next days, six daily acquisition trials of 15 minutes were performed with all subjects in random order. A fixed interval cued reward schedule of 30 seconds (FI 30) was applied whereby the cue lights were turned on every 25 seconds for five seconds and two food pellets were delivered immediately at the onset. After the last day of acquisition the aged and adult subjects were each assigned to one of two groups, either receiving regular drinking water (vehicle) or CORT-enriched water (CORT; 6$9 \mathrm{mg} / \mathrm{day} / \mathrm{kg}$, depending on the amount of water intake/rat). For the next 23 days, the animals received fresh drinking water daily and the water intake was measured for each $24 \mathrm{hr}$-period to control the intake of CORT. Re-acquisition trials were performed on every 3rd day. After 23 
days of treatment and re-training, extinction trials were run daily for five days under continuous food deprivation and treatment. Here, the cue lights were presented for 15 minutes with an FI 30 free reward schedule as well, but without coincident food delivery (Komorowski et al., 2012; Huston et al., 2016). The following variables were recorded as: a.) EID-related: mean 'distance to the food cup' and 'goal tracking' (direct contact with the food cup by entering or touching it); b.) Re-learning-related: 'number of beam breaks' in the food cup, 'latency between cue light onset and first consecutive beam break', 'sign tracking' (glances/rears towards the cue lights), and residence within the withdrawal compartment of the chamber, and c.) 'horizontal activity' (distance moved) and 'vertical activity' (rearing). The withdrawal compartment was situated in the latter part of the chamber and therefore offered the possibility to withdraw. Goal tracking (direct contact with the food cup by entering or touching it) and sign tracking (glances/rears towards the cue lights) were recorded manually by an experienced and blinded rater.

\section{Restraint stress and blood analysis}

In order to test for responsiveness of the hypothalamus-pituitary-adrenal (HPA)-axis, a restraint stress procedure was carried out 3 weeks after extinction. Treatment was continued until then. Immediately after a baseline blood sample $(\mathrm{t}=0)$, animals were put into restrainers (Harvard Apparatus ${ }^{\circledR}$ ), where they remained for 10 minutes. The second sample was taken in the restrainer after ten minutes $(t=10)$ and further samples in a single cage after $60(t=60), 120$ $(\mathrm{t}=120)$ and 180 minutes $(\mathrm{t}=180)$. Blood samples were taken by tail incision (Fluttert et al.,

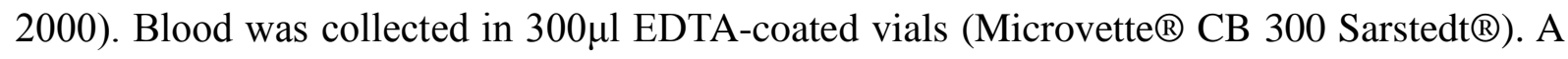
red light was mounted over the restrainers to provide warmth. Each subject was taken to the lab individually and remained in a single cage to recover for two hours after the last sampling. Samples were centrifuged at $4^{\circ} \mathrm{C}$ for ten minutes with a speed of $4000 \mathrm{rpm}$. The plasma was separated and stored by $-80^{\circ} \mathrm{C}$. For plasma hormone analysis, only animals with sufficient 
samples could be used, resulting in an $n=7-10$ /group. Adrenocorticotropic hormone (ACTH) plasma concentrations were analysed by chemo-iluminescence immunometric assay (Immulite ${ }^{\circledR} 2000$ ACTH, Siemens, Erlangen, Germany) and CORT plasma concentrations by ELISA (IBL International, Hamburg, Germany).

\section{Drugs \& Application}

Animals were treated chronically for 28 days until the last day of extinction. Before the onset of the treatment, the daily water intake of the rats during food deprivation was measured for two weeks. Water bottles were weighed and replaced every 24 hours during the experiment. Animals were housed in groups, so the total intake of each cage was divided by the number of rats to estimate the individual intake of water. Adult rats had a consistently higher mean water intake per day than aged rats. To ensure a daily CORT intake of $\sim 6-9 \mathrm{mg} / \mathrm{kg}$ for both, aged and adult rats, two distinct water solutions were produced, adapted to the different water intake. For the aged rats, a concentration of $125 \mu \mathrm{g} / \mathrm{ml}$ was chosen and $50 \mu \mathrm{g} / \mathrm{ml}$ for the adult rats. Corticosterone hemisuccinate (Tocris $\left.{ }^{\circledR}\right)$ was dissolved in distilled water adjusted to an alkaline $\mathrm{pH}$ of 13 with $4 \mathrm{M}$ sodium hydroxid (NaOH; Gourley \& Taylor, 2009). After dissolution, the $\mathrm{pH}$-value was equalized back to $7-7,4$ by $25 \%$ hydrochloric acid.

\section{Statistics}

Data were analysed as means $\underline{ \pm}$ S.E.M. Effects of age and treatment were analysed by twoway-ANOVA using baseline values as a covariate when appropriate. Pre-planned comparisons for single groups and time-points used t-tests. For effect size estimation of significantly different time points we calculated Cohen's d. A p-value $<0.05$ was considered significant. 


\section{Results}

In the re-acquisition trials, the distance to the food cup was higher in aged than in adults rats $\left(\mathrm{F}_{1,50}=10.94, \mathrm{p}<0.01\right)$, but was not affected by CORT or an interaction $(\mathrm{p}>0.05)$. During extinction, the distance increased $\left(\mathrm{F}_{4,196}=16.81, \mathrm{p}<0.001\right.$; Fig. $\left.2 \mathrm{~A}\right)$, but no main effects of age or CORT were found $(\mathrm{p}>0.05)$. Pre-planned comparisons showed a significant enhancement of the distance after CORT in adult $(\mathrm{d}=0.90)$ and aged animals $(\mathrm{d}=0.65)$ on extinction day 2 $(\mathrm{p}<0.05)$. Adult rats treated with CORT, however, showed a shorter distance to the food cup than controls on day $5(\mathrm{p}<0.01 ; \mathrm{d}=1.41$; Tab.1).

In the re-acquisition trials, goal tracking did not differ in frequency or duration with respect to the treatment $(p>0.05)$, but as a function of age (frequency: $F_{1,50}=45.1, p<0.001$; duration: $\left.F_{1,50}=16.32, p<0.001\right)$, with no interaction $(p>0.05)$. Aged rats showed a lower number $(\mathrm{p}<0.001)$ and shorter duration compared to adults $(\mathrm{p}<0.001)$. During extinction, the frequency of goal tracking decreased $\left(\mathrm{F}_{4,196}=164.73\right.$, $\mathrm{p}<0.001$; Fig. 2B). This effect was agedependent $\left(\mathrm{F}_{1,49}=267.45, \mathrm{p}<0.001\right)$ and affected by CORT $\left(\mathrm{F}_{1,49}=6.39, \mathrm{p}<0.05\right)$. Pre-planned comparisons showed that the frequency was lower in aged than in adult rats on each test day ( $\mathrm{p}<0.001$, all days). CORT reduced the frequency in adult rats on day $2(\mathrm{p}=0.001 ; \mathrm{d}=1.41)$ and in aged rats on day $5(\mathrm{p}<0.05 ; \mathrm{d}=1.25)$. The duration of goal tracking declined during extinction $\left(\mathrm{F}_{4,196}=56.5\right.$, $\mathrm{p}<0.001$; Fig. $\left.2 \mathrm{C}\right)$. There was an effect of age $\left(\mathrm{F}_{1,49}=31.52, \mathrm{p}<0.001\right)$, but not treatment or interaction effect $(\mathrm{p}>0.05)$. On each day of extinction, aged rats spent less time goal tracking than adults $(\mathrm{p}<0.05$, all days). CORT had no significant effect nor interacted with age $(\mathrm{p}>0.05)$. Pre-planned comparisons revealed that CORT further reduced the duration of goal tracking in aged rats on day $5(\mathrm{p}<0.001 ; \mathrm{d}=1.57)$.

On the last day of re-acquisition, neither the latency nor the total number of beam breaks was influenced by CORT ( $>0.05$; data not shown). Higher age did not have an impact on the latency $(p>0.05)$, but reduced the number of beam breaks $\left(F_{1,50}=25.53, p<0.001\right)$, with no interaction between age and treatment $(\mathrm{p}>0.05)$. During extinction, there was an increase in 
the latency of beam breaks $\left(\mathrm{F}_{4,196}=127.19, \mathrm{p}<0.001\right)$ and an effect of age $\left(\mathrm{F}_{1,49}=76.33\right.$, $\mathrm{p}<0.001$ ), with aged rats showing longer latencies to look into the food cup than adults ( $\mathrm{p}<0.001$; all days). CORT had no impact on the latency; neither was there an interaction with age $(p>0.05)$. The number of beam breaks decreased during extinction $\left(\mathrm{F}_{4,196}=10.06\right.$, $\mathrm{p}<0.001)$. An effect of age $\left(\mathrm{F}_{1,49}=45.16, \mathrm{p}<0.001\right)$ and an interaction with CORT was detected $\left(\mathrm{F}_{1,49}=6.67, \mathrm{p}<0.001\right)$, but no effect of CORT alone $(\mathrm{p}>0.05)$. Pre-planned comparisons indicated that the number of beam breaks was overall lower in the aged animals $(\mathrm{p}<0.05$; all days).

In the re-acquisition trials, sign tracking did not differ in frequency or duration with respect to the treatment $(\mathrm{p}>0.05)$. However, aged rats performed sign tracking less often $\left(\mathrm{F}_{1,50}=10.54\right.$, $\mathrm{p}=0.002)$ and for shorter duration $\left(\mathrm{F}_{1.50}=8.07, \mathrm{p}=0.006\right)$ than adults (Fig. 3A). During extinction, the frequency declined significantly $\left(\mathrm{F}_{4,196}=20.1, \mathrm{p}<0.001\right)$, which was affected by age $\left(\mathrm{F}_{1,196}=59.9, \mathrm{p}<0.001\right)$, but not by CORT or an interaction $(\mathrm{p}>0.05)$. Aged rats performed less sign tracking than adult rats at all time points $(\mathrm{p}<0.001)$. Pre-planned comparisons revealed that CORT reduced frequency of sign tracking in aged rats on day $2(\mathrm{p}=0.034$; $\mathrm{d}=0.82)$. The duration of sign tracking changed during extinction $\left(\mathrm{F}_{4,196}=5.51, \mathrm{p}<0.001\right)$. This effect was age-dependent $\left(\mathrm{F}_{1,49}=31.02, \mathrm{p}<0.001\right)$, but not influenced by CORT or an interaction $(\mathrm{p}>0.05)$.

In the re-acquisition trials, aged rats entered the withdrawal compartment less frequently than adult rats $\left(\mathrm{F}_{1,50}=16.82, \mathrm{p}<0.05\right)$, whereas the time spent in this compartment was not affected by age $(p>0.05)$. There was no effect of CORT or interaction with age $(p>0.05$; data not shown). During extinction, the number of entries into the operant compartment did not change over time ( $p>0.05$; data not shown). There was an effect of age $\left(\mathrm{F}_{1,49}=83.09, \mathrm{p}<0.001\right)$. There was a lower frequency of entries into the compartment during all days of extinction in aged compared to adult rats ( $\mathrm{p}<0.001$; for all time points). During extinction, the time spent in this 
compartment rose significantly $\left(\mathrm{F}_{4,196}=13.74, \mathrm{p}<0.001\right)$, independent of age or treatment $(\mathrm{p}>0.05)$

In the re-acquisition trials, aged rats moved less distance compared to adult rats $\left(\mathrm{F}_{1,50}=61.41\right.$, $\mathrm{p}<0.001)$, which was not affected by the treatment or an interaction ( $\mathrm{p}>0.05$; data not shown). Rearing behaviour differed significantly in frequency $\left(\mathrm{F}_{1,50}=32.66, \mathrm{p}<0.001\right)$ and duration $\left(\mathrm{F}_{1,50}=5.48, \mathrm{p}=0.023\right)$ as a function of age, but not of the treatment or interaction $(\mathrm{p}>0.05)$. Aged rats reared less often and with shorter duration than adult rats $(\mathrm{p}<0.001)$. During extinction, there was a significant decrease of the total distance moved for all groups $\left(\mathrm{F}_{4,196}=6.0, \mathrm{p}<0.001\right)$, which was not affected by CORT $(\mathrm{p}>0.05)$. However, age had a significant main effect $\left(\mathrm{F}_{1,49}=94.07, \mathrm{p}<0.001\right)$, with aged animals walking less distance than adult animals ( $<<0.001$; for all time points). During extinction, there was a significant decrease in the frequency of rearing $\left(\mathrm{F}_{4,196}=21.38, \mathrm{p}<0.001\right)$, which was age-dependent $\left(\mathrm{F}_{1,49}=188.61, \mathrm{p}<0.001\right)$, but not affected by CORT $(\mathrm{p}>0.05)$. Also the duration of rearing changed over time $\left(\mathrm{F}_{4,196}=4.68, \mathrm{p}=0.001\right)$. This effect was age-dependent $\left(\mathrm{F}_{1,49}=115.61\right.$, $\mathrm{p}<0.001)$. Pre-planned comparisons revealed shorter duration of rearing in aged compared to adult rats for each day of extinction $(\mathrm{p}<0.05$; for all time points). Subsequently, the mean duration of a single rear was calculated (Fig. 3B). The duration increased over time $\left(\mathrm{F}_{4,196}=12.53\right.$, $\left.\mathrm{p}<0.001\right)$. This was influenced by age $\left(\mathrm{F}_{1,49}=20.14, \mathrm{p}<0.001\right)$, with aged rats rearing for longer periods of time than adult rats $(\mathrm{p}<0.05)$. CORT tended to reduce this parameter $\left(\mathrm{F}_{1,49}=3.95, \mathrm{p}=0.053\right)$.

At baseline, chronic CORT treatment reduced plasma CORT levels $\left(\mathrm{F}_{1,48}=4.6, \mathrm{p}<0.05\right)$ with no effect of age or interaction ( $\mathrm{p}>0.05$; Fig. 4A-B). Pre-planned comparisons showed a significant decrease after CORT only in aged $(\mathrm{p}<0.05 ; \mathrm{d}=1.26)$, but not in adult rats $(\mathrm{p}>0.05)$. Restraint stress increased CORT level $\left(\mathrm{F}_{3,126}=3.54, \mathrm{p}<0.05\right)$. However, CORT treatment reduced the CORT response $\left(\mathrm{F}_{1,42}=34.69, \mathrm{p}<0.001\right)$, while age had no effect $(\mathrm{p}>0.05)$. Pre- 
planned comparisons showed that adult CORT-treated subjects had lower levels of CORT in the plasma than the control groups $10 \min (\mathrm{p}<0.01 ; \mathrm{d}=1.47)$ and $180 \min (\mathrm{p}<0.05 ; \mathrm{d}=1.59)$ after start of restraint. Aged CORT-treated subjects had lower levels of CORT in the plasma $10,60,120 \min (\mathrm{p}<0.001 ; \mathrm{d}=2.54,1.96,1.89)$ and $180 \min (\mathrm{p}<0.01: \mathrm{d}=1.42)$ after start of restraint (Fig. 4A-B).

At baseline, chronic CORT treatment reduced plasma ACTH levels $\left(\mathrm{F}_{1,48}=8.92, \mathrm{p}<0.01\right)$ with no effect of age or interaction ( $p>0.05$; Fig. 4C-D). Pre-planned comparisons showed a decrease after CORT only in aged $(\mathrm{p}<0.001 ; \mathrm{d}=1.74)$, but not in adult rats $(\mathrm{p}>0.05)$. Visual inspection of the data suggests that restraint stress increased plasma ACTH level, which, however, failed to reach significance $(\mathrm{p}>0.05)$. There was an effect of age $\left(\mathrm{F}_{1,33}=9.96\right.$, $p<0.01)$, but no effect of the CORT treatment or interaction $(p>0.05)$. 


\section{Discussion}

The re-acquisition of an operant task, originally learnt under drug-naïve conditions, was not significantly impaired by the chronic treatment with CORT. Other studies had shown that reward-learning is impaired after a chronic CORT treatment (Olausson et al., 2013). This discrepancy may have been due to animals initially learning the task in a drug-free state in this study. Here, aged rats showed a reduced performance in nearly all behavioral parameters after acquisition (Tab.1). Nevertheless, it cannot be ruled out that present results may be due to a decline in general locomotor activity in the aged rats (Stanford et al., 2002) rather than differences in learning ability per se.

The 'distance to the food cup' increased and 'goal tracking' decreased significantly over the extinction trials in all animals, indicating that an EID-related phenotype appears in adult as well as aged rats in this model. Treatment with antidepressants significantly reduced EID in adult and aged rats in the water maze and in an operant chamber (Schulz et al., 2007, Komorowski et al., 2012). In accordance with our main hypothesis, CORT treatment enhanced the distance to the former site of reward-delivery and avoidance of goal tracking during extinction predominantly in aged rats.

The latency and number of beam breaks increased and decreased, respectively, and 'sign tracking' decreased over the extinction trials in all animals, in adult as well as aged animals. While aging reduced the resistance to extinction, CORT treatment had no effect in adult or aged animals. Together, these findings suggest that stress enhances the negative emotional component of extinction particularly in the aged organism.

Aged rats chronically treated with CORT showed attenuated plasma levels of CORT and ACTH. The application of exogenous CORT promotes the negative feedback inhibition of the HPA-axis and hence decreases the levels of endogenous CORT/ACTH (Sapolsky et al., 1986b). After restraint, all animals exhibited a significant increase in CORT. This was blunted in both age groups by chronic CORT treatment. The chronic treatment presumably inhibited 
the HPA-axis and suppressed the release of endogenous CORT with a blunted response to restraining (Johnson et al., 2006). Present findings suggest that chronic stress may reduce basal HPA-axis activity predominantly in the aged organism, while the reduced activation by acute stress is independent of age. This may suggest that adaptations of HPA-axis activity could be responsible for enhanced EID during extinction in the aged organism.

It should be noted that the application of CORT via drinking water might be prone to variations in the daily uptake of CORT. Since animals were not housed alone during the treatment period but in cages of 2-4 rats, the exact individual uptake of water and CORT could not be quantified. However, the method is non-invasive and therefore does not subject the animals to further stress. Additionally, single housing is a form of social isolation that might alter the animals` behavior to an unknown extent.

Locomotor behavior differed, as expected, as a function of age. Adult rats traveled a larger distance and reared more often than aged rats (Stanford, Currier \& Gerhardt, 2002). The distance moved was not affected by the CORT treatment and neither was the frequency and duration of rearing. These findings are supportive of other reports that do not show an effect of chronic CORT treatment on horizontal and vertical activity, even in old rats (Brotto, Gorzalka \& Barr, 2001). The mean duration of a single rearing unit increased over the extinction trials. The duration of a unit was higher in aged than in adult rats. CORT tended to decrease the duration of a single rear. The duration and frequency of rearing are also considered to be markers of non-selective attention (NSA), especially when directed towards environmental stimuli (Aspide et al., 1998; 2000). Therefore, a reduction in the duration of single rearing units by the CORT treatment might indicate a shift in NSA.

Overall, the present findings further support the validity of the EID model. Resistance to extinction was lower in the aged than adult rats. Treatment with CORT increased EID-like behaviors predominantly in the aged rats together with more pronounced decline in HPA-axis 
activity. Thus, this animal model of EID may be particularly useful to serve as a model for geriatric depression.

\section{Acknowledgements}

Supported by the German National Science Foundation Grants Hu 306/27-2 and Mu 2789/81, the Research Training Group GRK 1033-2 and Heisenberg Fellowship SO 1032/5-1 from the Deutsche Forschungsgemeinschaft to M.A.d.S.S. We thank U. Lehnert for the analysis of the hormone-assays. 


\section{References}

Bruce ML (2002) Psychosocial risk factors for depressive disorders in late life. Biological Psychiatry, 52, 175-184.

Huston JP, van den Brink J, Komorowski M, Huq Y, Topic B (2012) Antidepressants reduce extinction-induced withdrawal and biting behaviors: A model for depressive-like behavior. Neuroscience, 210, 249-257.

Huston JP, de Souza Silva MA, Komorowski M, Schulz D, Topic B (2013) Animal models of extinction-induced depression: Loss of reward and its consequences. Neuroscience and Biobehavioral Reviews, 37(9), 2059-2070.

Huston JP, Kornhuber J, Mühle C, Japtok L, Komorowski M, Mattern C, Reichel M, Gulbins E, Kleuser B, Topic B, De Souza Silva MA, Müller CP (2016) A sphingolipid mechanism for behavioral extinction. Journal of Neurochemistry, 137, 589-603.

Johnson SA, Fournier NM, Kalynchuk LE (2006) Effect of different doses of corticosterone on depression-like behavior and HPA axis responses to a novel stressor. Behavioral Brain Research, 168, 280-288.

Kendler KS, Karkowski LM, Prescott CA (1999) Causal relationship between stressful life events and the onset of major depression. The American Journal of Psychiatry, 156, 837841.

Komorowski M, Huston JP, Klingenhegel I, Paulat J, Sackers J \& Topic B. (2012) Distance from source of reward as a marker for extinction-induced "despair": Modulation by the antidepressants clomipramine and citalopram. Neuroscience, 223, 152-162.

Kraaij V, Arensman E, Spinhoven P (2002) Negative life events and depression in elderly persons: a meta-analysis. Journal of Gerontology: Psychosocial Sciences, 57(B), 87-94. 
Lewinsohn PM (1974) A behavioral approach to depression. In R.J. Friedman, \& M.M. Katz (Eds.), The Psychology of Depression: Contemporary Theory and Research (pp. 157-85). New York: Wiley.

Olausson P, Kiraly DD, Gourley SL, Taylor JP (2013) Persistent effects of chronic exposure to corticosterone on reward related learning and motivation in rodents. Psychopharmacology, 225(3), 569-577.

Papini MR, Dudley RT (1997) Consequences of surprising reward omission. Reviews of General Psychology, 1, 175-197.

Papini MR (2003) Comparative psychology of surprising nonreward. Brain, Behavior and Evolution, 62, 83-95.

Sapolsky RM, Krey LC, McEwen BS (1986) The neuroendocrinology of stress and aging: The glucocorticoid cascade hypothesis. Endocrine Reviews, 7, 287-301.

Schulz D, Topic B, de Souza Silva MA, Huston JP (2004) Extinction-induced immobility in the water maze and its Neurochemical concomitants in aged and adult rats: A possible model for depression? Neurobiology of Learning and Memory, 82, 128-141.

Schulz D, Buddenberg TE, Huston JP (2007) Extinction-induced "despair" in the water maze, exploratory behavior and fear: Effects of chronic antidepressant treatment Neurobiology of Learning and Memory, 87, 624-634.

Stanford JA, Currier TD, Gerhardt GA (2002) Acute locomotor effects of fluoxetine, sertraline, and nomifensine in young versus aged Fischer 344 rats. Pharmacology, Biochemistry and Behavior, 71, 325-332.

Todd TP, Vurbic D, Bouton ME (2014) Behavioral and neurobiological mechanisms of extinction in Pavlovian and instrumental learning. Neurobiology of Learning and Memory, 108, 52-64. 
Topic B, Huston JP, Nametskova K, Zhu SW, Mohammed AH, Schulz D (2008a) Extinctioninduced "despair" in aged and adult rats: Links to neurotrophins in frontal cortex and hippocampus. Neurobiology of Learning and Memory, 90, 519-526.

Topic B, Oitzl MS, Meijer OC, Huston JP, de Souza Silva MA (2008b) Differential susceptibility to extinction-induced despair and age-dependent alterations in the hypothalamic-pituitary-adrenal axis and neurochemical parameters. Neuropsychobiology, $58,138-153$.

Wittchen HU, Jacobi F, Rehm J, Gustavsson A, Svensson M, Jönsson B, Olesen J, Allgulander C, Alonso J, Faravelli C, Fratiglioni L, Jennum P, Lieb R, Maercker A, van Os J, Preisig M, Salvador-Carulla L, Simon R, Steinhausen H-C (2011) The size and burden of mental disorders and other disorders of the brain in Europe 2010. European Neuropsychopharmacology, 21(9), 655-679. 


\section{Figure and table legends}

Figure 1 The two-compartment operant chamber.

Figure 2 The effects of age (adult vs. aged) and corticosterone (CORT) treatment on extinction-induced depression (EID)-related behaviors during the extinction of a cued fixedtime free-reward delivery task. Results show mean \pm SEM. (A) Distance from the food cup and (B) goal tracking frequency and (C) goal tracking duration reflect EID measures. $\left({ }^{*} \mathrm{p} \leq 0.05 ; * * \mathrm{p} \leq 0.01 ; * * * \mathrm{p}<0.001 ;\right.$ vs. vehicle (veh)).

Figure 3 The effects of age (adult vs. aged) and corticosterone (CORT) treatment on activityrelated behaviors during the extinction of a cued fixed-time free-reward delivery task. Results show mean \pm SEM. (A) Sign tracking and (B) Mean duration of a single rearing episode $(* \mathrm{p} \leq 0.05$ vs vehicle $($ veh $))$.

Figure 4 The effects of age (adult vs. aged) and corticosterone (CORT) treatment on (A-B) plasma CORT level, and (C-D) plasma ACTH levels after the treatment and physical stress in a 10 min (grey bar) restraint stress procedure $\left({ }^{*} \mathrm{p} \leq 0.05 ;{ }^{*} \mathrm{p} \leq 0.01 ; * * * \mathrm{p}<0.001\right.$ vs vehicle (veh)).

Table 1 Table 1 The effects of age (adults vs aged) and corticosterone treatment (CORT) on the acquisition and extinction of a cued fixed-time free-reward delivery task in adult and aged rats (EID - parameters related to extinction-induced depression; RL - parameters related to re-learning during extinction according to ${ }^{1}$ Komorowski et al., 2012 and ${ }^{1,2}$ Huston et al., 2013, 2016; Acquisition: + enhanced, - reduced, or "o" no effect on acquisition of behavior; Extinction: $\uparrow$ enhanced extinction, $\downarrow$ reduced extinction, --- no effect on extinction). 


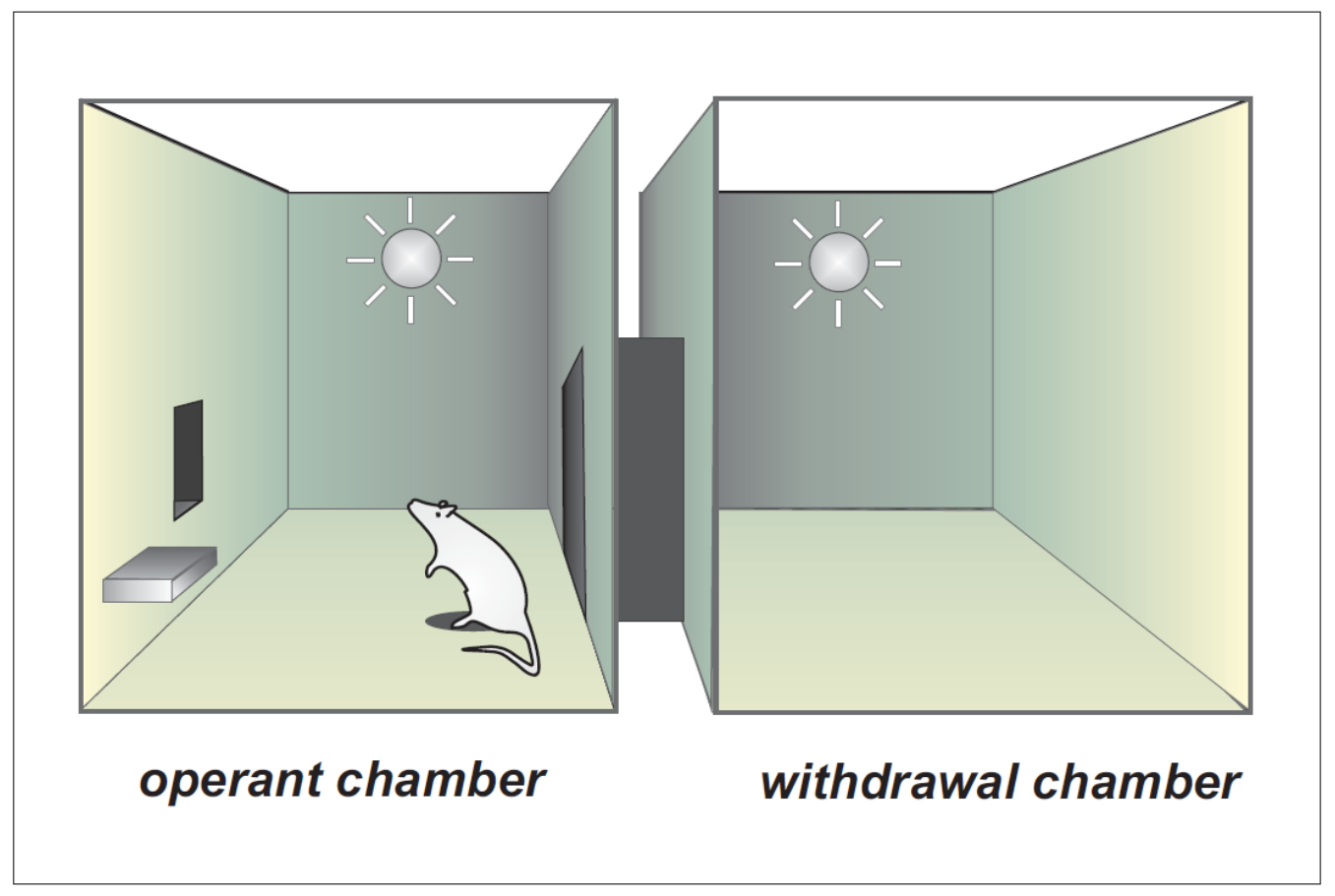

Figure 1 


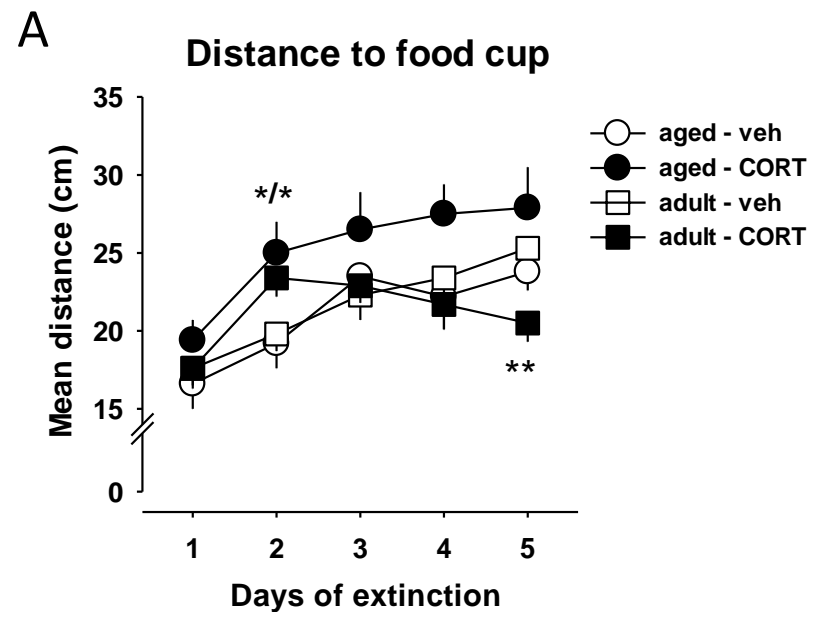

B Goal tracking - frequency
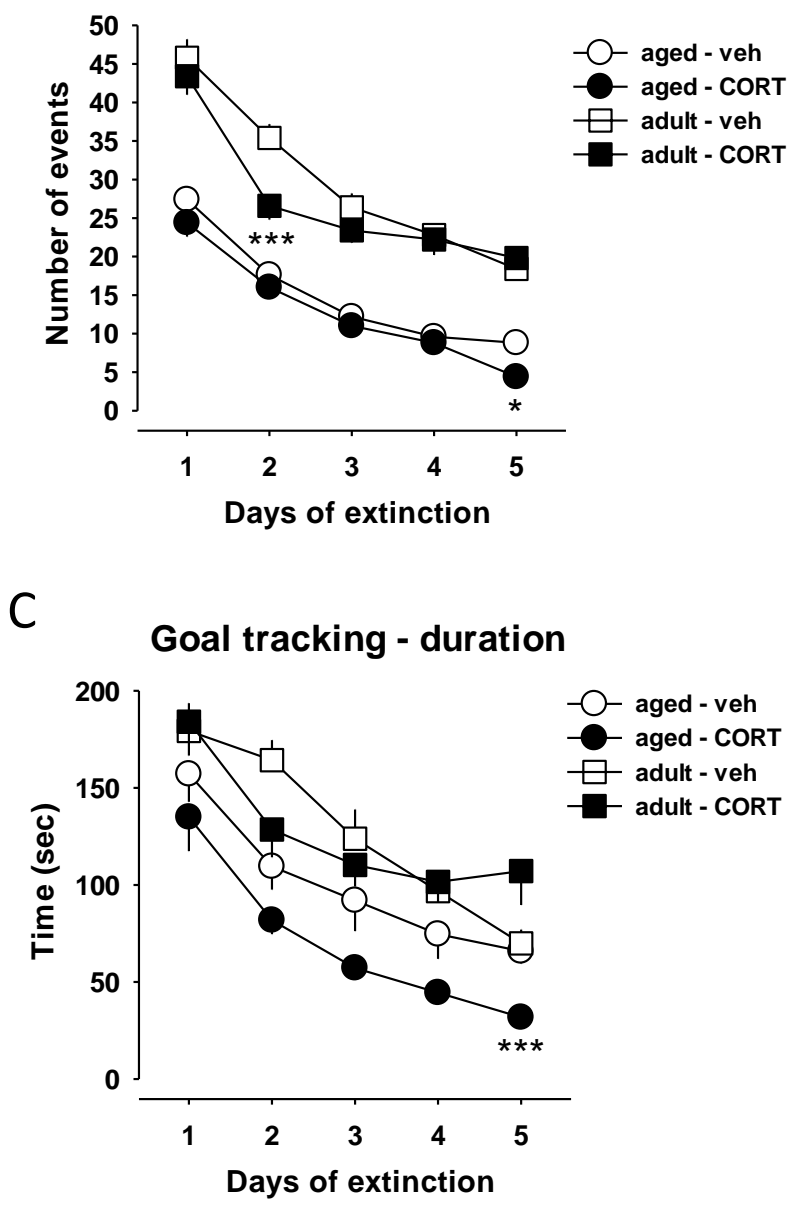

Figure 2 
A

Sign tracking

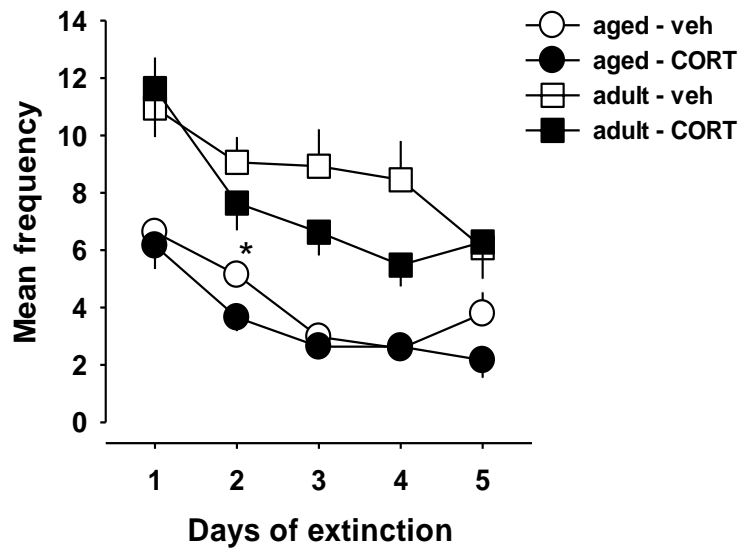

B

Rearing

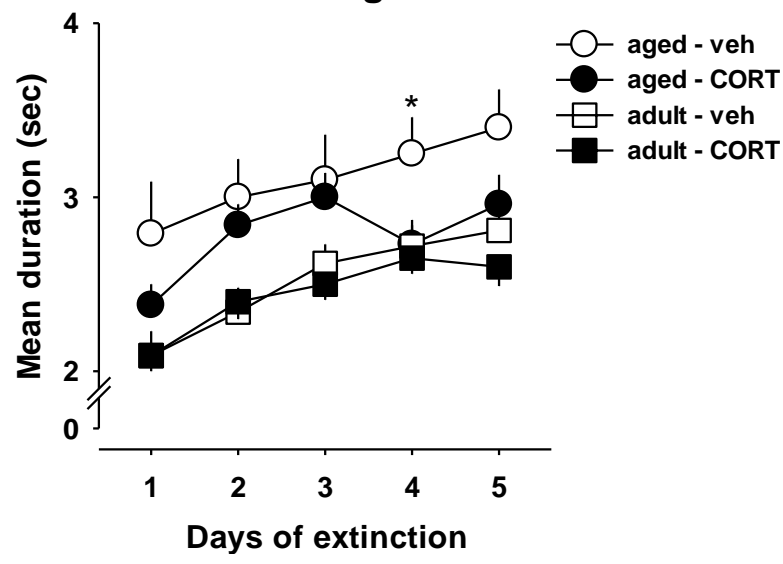

Figure 3 
A

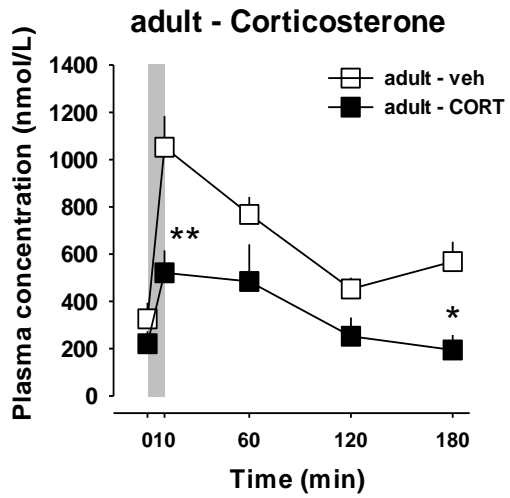

C

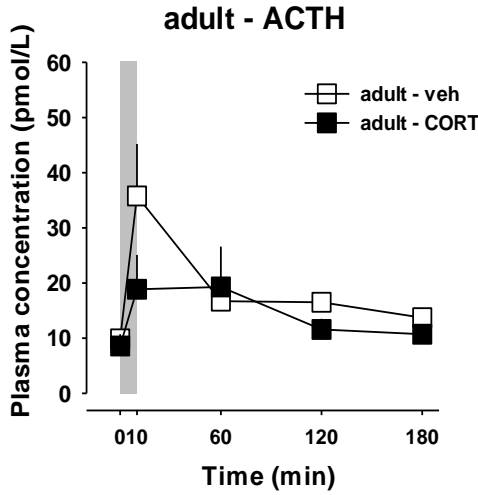

B

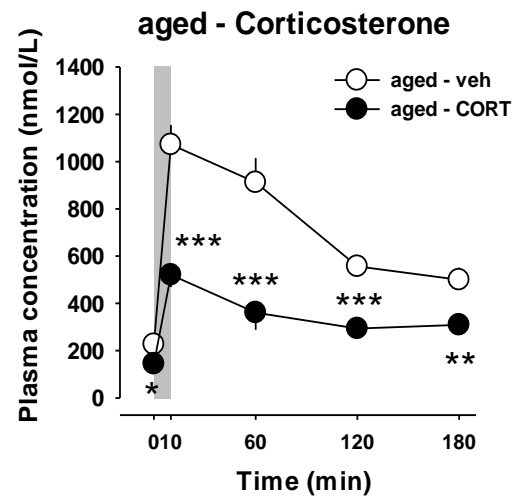

D

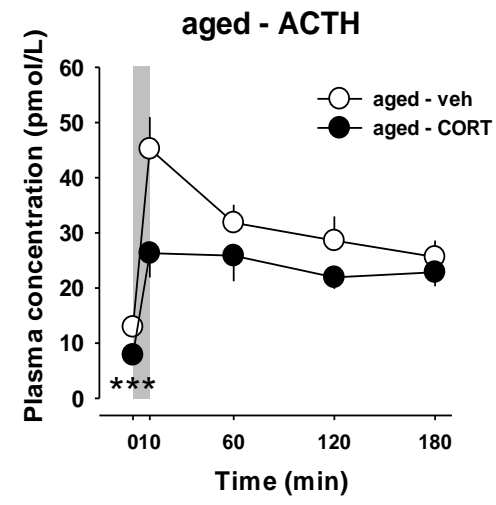


Table 1 The effects of age (adults vs aged) and corticosterone treatment (CORT) on the acquisition and extinction of a cued fixed-time free-reward delivery task in adult and aged rats (EID - parameters related to extinction-induced depression; RL - parameters related to re-learning during extinction according to ${ }^{1}$ Komorowski et al., 2012 and ${ }^{1,2}$ Huston et al., 2013, 2016; Acquisition: + enhanced, - reduced, or "o" no effect on acquisition of behavior; Extinction: $\uparrow$ enhanced extinction, $\downarrow$ reduced extinction, --- no effect on extinction).

\begin{tabular}{|c|c|c|c|c|c|c|c|c|}
\hline & \multirow[t]{2}{*}{ EID $^{1}$} & \multirow[t]{2}{*}{$\mathbf{R} \mathbf{L}^{2}$} & \multicolumn{3}{|c|}{ Acquisition } & \multicolumn{3}{|c|}{ Extinction } \\
\hline & & & Age & CORT & $\mathrm{AxC}$ & Age & CORT & $\mathrm{AxC}$ \\
\hline Distance to the food cup & $\mathrm{X}$ & & - & o & o & --- & --- & CORT: aged $\uparrow /$ adult $\uparrow / \downarrow$ \\
\hline Goal tracking - frequency & $X$ & & - & o & o & $\uparrow$ & --- & CORT: aged $\uparrow /$ adult $\uparrow$ \\
\hline Goal tracking - duration & $\mathrm{X}$ & & - & o & o & $\uparrow$ & --- & CORT: aged $\uparrow$ \\
\hline Latency of beam breaks & & $X$ & o & o & o & $\uparrow$ & --- & \\
\hline Number of beam breaks & & $\mathrm{X}$ & - & o & o & $\uparrow$ & --- & --- \\
\hline Withdrawal zone entries & & & - & o & o & --- & --- & --- \\
\hline Withdrawal zone time & & & o & o & o & --- & --- & --- \\
\hline Sign tracking - frequency & & & - & o & o & $\uparrow$ & --- & CORT: aged $\uparrow$ \\
\hline Sign tracking - duration & & & - & o & o & $\uparrow$ & --- & --- \\
\hline Horizontal activity & & & - & o & o & $\uparrow$ & --- & --- \\
\hline Rearing - frequency & & & - & o & o & $\uparrow$ & --- & --- \\
\hline Rearing - duration & & & - & o & o & $\uparrow$ & --- & --- \\
\hline
\end{tabular}

